\title{
Circuit
}

Musiques contemporaines

\section{Gradus ad Infernum}

Entrevue avec Ferdinand Larven Niemantz, " penseur de la

musique " et auteur de Musical Compositions of the Century

\section{Gradus ad Infernum}
Interview with Ferdinand Larven Niemantz, "Thinker in Music" and Author of Musical Compositions of the Century

\section{John Rea}

Volume 9, numéro 2, 1998

Carte blanche à Bouliane et Rea

URI : https://id.erudit.org/iderudit/902227ar

DOI : https://doi.org/10.7202/902227ar

Aller au sommaire du numéro

Éditeur(s)

Les Presses de l'Université de Montréal

ISSN

1183-1693 (imprimé)

1488-9692 (numérique)

Découvrir la revue

Citer ce document

Rea, J. (1998). Gradus ad Infernum : entrevue avec Ferdinand Larven Niemantz, " penseur de la musique " et auteur de Musical Compositions of the Century.

Circuit, 9(2), 9-24. https://doi.org/10.7202/902227ar
Résumé de l'article

Cette entrevue imaginaire, accordée à l'auteur par ce maître penseur de la musique, soulève en maints endroits la problématique du simulacre de la vérité masquée, du faire-semblant et de la supercherie que pourrait être la postmodernité. L'écrivain autrichien se penche sur une topologie moderniste parcourant les forêts, les prairies, les catégories et les valeurs socio-musicales qui caractérisent cette fin de millénaire. d'utilisation que vous pouvez consulter en ligne. 


\title{
Gradus ad Infernum
}

\author{
Entrevue avec Ferdinand Larven Niemantz, \\ " penseur de la musique " et auteur de \\ Musical Compositions of the Century \\ John Rea
}

Ma première rencontre avec l'intellectuel autrichien Ferdinand Larven Niemantz remonte à l'époque où j'étais inscrit au doctorat en composition à l'université Princeton, il y a près de trente ans. À la suggestion d'un ami, j'étais auditeur libre à l'un des cours qu'il donnait devant une de ces immenses classes de niveau collégial. Niemantz était alors un nouveau professeur de littérature comparée, domaine qui intéressait énormément les jeunes à la fin des années 1960. Je ne tardai pas à assister à son cours de façon régulière ainsi qu'à participer après la classe à certaines discussions suscitées par ses remarques. Il avait l'habitude d'entraîner ses étudiants à un café ou à une taverne sympathique, et là nos conversations couvraient une grande variété de sujets. A ma grande surprise, la musique était un sujet privilégié. Mais cela éveillait plutôt chez moi le soupçon : il semblait en savoir beaucoup plus que qu'il ne le laissait paraître. II y avait aussi le fait inhabituel qu'il m'appelait toujours «le Canayen », référence que j'étais trop naif pour apprécier à sa juste valeur jusqu'à ce qu'il en explique un jour le sens au Torontois de naissance que je suis. Ce qu'il connaissait du Canada se limitait à de nombreuses visites à Québec et à Montréal ; au cours des deux décennies qui suivirent, il visita la plupart des grandes villes du pays, surtout celles de l'Ontario. II continue de voyager beaucoup, surtout vers des destinations éloignées, quoique moins souvent que dans le passé.

Je suis rentré au Canada peu après la fin de mes études. C'est à cette époque que des changements aussi rapides que tumultueux balayèrent les campus universitaires américains, changements qui eurent des conséquences désastreuses pour Niemantz : les inscriptions à ses cours amorcèrent une chute vertigineuse. À tel point qu'après seulement cinq années d'enseignement, il remit brusquement sa démission en 1972. Ce bouleversement l'obligea à reconsidérer sa vie et ses travaux du fait que ses études - un doctorat en langues 
romanes de l'université Stanford lle titre de sa thèse était : Le Tremblement de terre de Lisbonne en 1755 et ses conséquences sur la poésie érotique portugaise) - sa formation, donc, ne l'avait aucunement préparé au désarroi psychologique qui accompagne le chômage.

Fort heureusement, le malheur de la littérature fit le bonheur de la musique. Niemantz décida de retourner à ses premières amours, à savoir la discipline à laquelle son père l'avait formé mais qu'il avait refusé d'exercer une fois adulte : la composition musicale. Non pas comme dans I' "art de la...", mais plutôt comme dans le "théâtre des » idées compositionnelles, l'euphémisme qu'il emploie aujourd'hui pour décrire son travail. II allait devenir, selon ses propres termes, "un penseur de la musique ». À l'âge de cinquante ans, il ajouta un autre doctorat, cette fois en musicologie à l'Université de Heidelberg (thèse : l'« idée " de Kant sur les idées en musique). À l'âge de soixante-dix ans, à la veille de faire paraître son premier ouvrage ${ }^{(1)}$, Ferdinand Larven Niemantz est aujourd'hui un homme au comble du bonheur malgré des signes de fatigue et de lassitude visibles sur son visage qui, je dois le souligner, n'est pas bronzé, fait exceptionnel pour qui a vécu près de soixante ans en Californie.

Notre entrevue, dont la première partie est reproduite ici, s'est déroulée chez moi, en anglais, pendant trois jours de l'automne 1996. M. Niemantz était de passage à Montréal pour assister au congrès international de la Société des technologies alternatives à valeur appliquée (STAVA). La voix de Niemantz résonne sans aucune trace d'accent. Son langage est naturel, parsemé de nombreuses locutions et jeux de mots non seulement en anglais, mais aussi en français, sa deuxième langue. Toujours spirituel et d'humeur égale, il fait encore montre à l'occasion de la vivacité et de la passion dont j'avais été témoin dans sa classe il y a trente ans. II manifeste souvent une ironie sèche et mordante. Nous avons parlé de beaucoup de choses, y compris de la musique canadienne, sujet avec lequel il a beaucoup d'affinités, bien que je qualifierais sa relation avec elle de complexe - comme avec tant d'autres musiques. Pour une foule de raisons, dont certaines apparaissent pendant l'entrevue, Niemantz me semble l'incarnation de l'intellectuel tragique de notre siècle, condition attribuable tant à son lieu d'origine qu'à son tempérament.

John Rea : À votre naissance, votre père Alwa était une "personne du troisième âge ", et vous ne l'avez pas beaucoup connu en dehors de vos cours de composition avec lui. Cela semblaitil inhabituel au jeune Viennois que vous étiez ?

Ferdinand Larven Niemantz: Non, pas vraiment. Notre maison était un lieu très vivant malgré l'âge avancé de mon père, mes parents faisant tout pour que leur enfant unique ne s'ennuie pas. J'avais hâte d'aller à mes leçons, mais après le divorce de mes parents il est devenu plus difficile de sauver les
(1) F. L. Niemantz, Musical Compositions of the Century, Pegasus (Palo Alto, 1997), 287 p. Deux CD-ROM sont inclus dans l'édition de luxe. Par pure coïncidence, The New York Library's Books of the Century, édité par E. Diefendorf, est paru en $1996 \mathrm{chez}$ Oxford University Press. 
apparences. Je voyais mon père moins souvent mais plus longtemps à la fois dans ses appartements à l'hôtel Excelsior. Les leçons se sont poursuivies de la sorte pendant quelque temps, puis j'ai appris que ma mère voulait partir avec moi pour l'Amérique immédiatement. On était en 1938.

J. R. : Votre père était un élève de Brahms, son dernier, m'avez-vous déjà dit, bien qu'il ne soit jamais devenu compositeur de musique de concert. Pourtant, il composait de la musique populaire. Le saviez-vous quand vous étiez jeune?

F. L. N. : Non. Ma mère m'a révélé ces choses à la fin des années 1940, juste avant de mourir, à l'époque où j'étudiais avec [Theodor Wiesengrund] Adorno à Los Angeles. On m'a toujours dit que mon père était un industriel, bien que je n'aie jamais vu une seule usine. Je n'ai jamais revu mon père après 1938.

Permettez-moi, je vous prie, de faire une correction mineure mais importante : père n'était pas compositeur de musique populaire. II a composé seulement une chanson! Notre fortune - ma fortune - repose entièrement sur cette bagatelle de 2 minutes 16 secondes qu'il avait écrite pour répondre à un défi lancé par l'une de ses maîtresses à la veille de la Grande Guerre. Mon père a dit à ma mère, à ce qu'il paraît, que cette vamp l'avait mis au défi d' "être comme l'autre Alwa ", allusion à [Thomas] Alva Edison, qui amassait alors une fortune avec ses nouvelles inventions, dont le phonographe. Peu après, père écrivit les paroles et la musique. La nouvelle technologie n'était pas encore arrivée en Autriche - je parle des disques 78 tours à deux côtés. Alors il a emprunté de l'argent pour faire un démo, comme on dit maintenant, sur un disque à un seul côté et, voilà, il a eu un « hit » qui lui a permis d'épouser ma mère, son grand amour. Elle l'a obligé plus tard, très sagement, à investir les redevances qui entraient à flots. Ces investissements lui ont apporté une fortune encore plus grande qui a survécu au krach de 1929, je ne sais comment. J'avais quatre ans à l'époque. Mon père n'a jamais abordé ce sujet avec moi ; je n'ai jamais entendu la chanson ni vu la partition. J'ai fait des efforts assidus pour la retracer, mais avec tous les bombardements et, bon, avec la disparition de mon père avant notre départ pour la Californie, tout a conspiré pour rendre cette tâche impossible. Peut-être a-til détruit des choses, ou ma mère? Je l'ignore.

J. R. : Comment s'intitulait la chanson ? A-til utilisé son nom véritable ?

F. L. N. : Mère m'a dit que le titre était Lurline, un diminutif affectueux pour Lorelei, la fille du Rhin dont les appas entraînaient les marins à la mort, la sirène qui cause les naufrages. Son nom de plume : Cric-Crac. Cela aussi rendait ma mère mal à l'aise, vous pensez bien. Le seul autre nom en musique populaire que je connaisse de cette époque (et qui rend un son tout aussi excentrique quand on le prononce) est celui de l'auteur des paroles d'une chanson napolitaine très connue, Musica proibita: Flick-Flock. 
J. R. : Vous croyiez tous les deux, si je comprends bien, que votre père Alwa a péri à Buchenwald, même si vous n'en avez jamais eu la preuve, ni de la part des Alliés ni de celle des Allemands. Pourquoi?

F. L. N. : Oui, c'est exact, mais le terme "croire » est trop fort. L'histoire est embrouillée parce que mère a été très affectée par la Guerre... Elle a dépéri très rapidement. Eł Adorno ne nous était d'aucun secours. II ne voulait rien savoir de mon père-compositeur-de-musique-populaire, qu'il considérait unmodernisch et dont il n'avait jamais entendu parler. II consacrait tout son temps, en compagnie de [Thomas] Mann, à leur fastidieux projet dodécaphonique, Faustus. Pourtant, le premier indice au sujet du destin de père est venu de nul autre qu'Adorno, qui m'a fait une peur terrible - n'effrayait-il pas un peu tout le monde? [rire pensif] - car c'est Wiesengrund qui m'a fait comprendre que le blason de famille de mère, du côté de sa mère, montrait l'image d'une forêt de hêtres. Je me suis rendu compte qu'il nous a dit cela en français afin d'adoucir le choc: cela signifie Buchenwald. Or père, voyez-vous, était un parent éloigné de ma grand-mère maternelle. Adorno nous a également rappelé que son second prénom à lui, Wiesengrund, qui signifie prairie, était en réalité le nom de famille de son père. Je ne peux l'expliquer, mais il nous a convaincus que c'était la seule façon de rendre compte de l'absence de père. J'étais jeune et influençable alors. C'est Wiesengrund qui m'a assommé peu de temps après avec un jeu de mots absolument inexcusable: Hêtre ou ne pas hêtre. J'ai su à ce moment qu'il fallait que ce soit vrai au sujet de Buchenwald, et je ne l'ai jamais oublié. Nous étions en 1947.

J. R. : Vous aviez vingt-deux ans, ce n'est pas si jeune.

F. L. N. : [Rire soudain.] Dit-il sur un ton d'arrogance aristocratique! Fort bien. Oui. Remarquez, je comprends cela. Mais vous ne devez pas oublier: je vénérais Adorno. Il était presque... presque comme un vrai père pour moi, un père avec qui je pouvais ressentir des pulsions œdipiennes, des pulsions que je n'avais pas connues avec l'homme âgé qu'était Alwa.

J. R. : Quels étaient les rapports de votre père avec le dramaturge Frank Wedekind ${ }^{(2)}$ ?

F. L. N. : Les rapports de Wiesengrund?

J. R. : Non, non, les rapports d'Alwa.

F. L. N. : Oh la la, quel lapsus !... Penser qu'encore aujourd'hui, ce... ce scélérat... ces effets psychologiques résiduels... Quoi qu'il en soit. Oui, Wedekind et mon père [Alwa] sont nés à Hanovre - le même jour - et ont fréquenté plus tard la même école primaire pendant une courte période de temps. [Pause.] (Saviez-vous que la future mère de Wedekind, je veux dire sa vraie mère, a vécu en Californie à l'époque de la ruée vers l'or ?...) Les deux hommes se
(2) Frank Wedekind (1864-1918), précurseur du Théâtre de l'Absurde et un des premiers dramaturges expressionnistes, est notamment l'auteur de Erdgeist 11895 ; Esprit de la terre) et de Die Büchse der Pandora 11904 ; La Boîte de Pandorel, mieux connu comme le cycle de Lulu. [N.d.É.] 
comportaient de façon conforme à l'éducation hanséatique abrégée que chacun avait reçue, mais ce fait ne les a pas empêchés de se brouiller, surtout après cette aventure sordide avec une gourgandine à Paris. Mère a dit que père n'a jamais pardonné à Wedekind d'avoir tout rapporté dans ses pièces, tous les événements, y compris son nom. Wedekind, disait-elle malicieusement, se réservait le rôle du père d'Alwa, le docteur Schön, un journaliste de second ordre, et il se fait tuer tôt dans I'histoire. Dans cette série de pièces, rappelezvous, Alwa Schön veut composer un opéra sur les événements qui surviennent dans l'existence démesurée de Lulu. L'obsessif-compulsif Wedekind se moquait de mon père, je pense, parce qu'il avait étudié avec Brahms le conservateur, un compositeur qui n'avait jamais écrit d'opéra. De toute façon, mère disait que Wedekind méritait de mourir jeune pour avoir vécu et écrit des épisodes aussi honteux. II avait cinquante-quatre ans.

D'une manière plus significative, les répercussions de cette tragique querelle se sont fait sentir bien des années plus tard, en 1937, et je m'en souviens avec autant de netteté que de tristesse. Des mois durant, à ce qu'il me semble, père en parlait sans arrêt à chaque pause-café pendant mes longues leçons à l'hôtel. Un pressentiment tangible l'habitait et il me communiquait une grande anxiété. Il devenait fou à l'idée que le nouvel opéra d'Alban Berg [Lu/u] serait bientôt créé à Zurich et, franchement, la date de la disparition de mon père correspond à son absence de l'hôtel un mois après la première représentation de l'opéra, au moment où une autre leçon avec lui était prévue. Nous avions commencé à préparer ma troisième composition pour orchestre. (Père disait souvent que Kandinsky avait raison d'appeler ses tableaux des compositions, vu l'agencement polyphonique de leurs timbres.) Cette nouvelle œuvre était censée refléter l'étude que nous avions effectuée sur ce qu'on appelle aujourd'hui une collection de hauteurs fixes, et leur effet de résonance sur ma polyphonie. Nous avions analysé d'innombrables œuvres d'Obrecht, de Josquin et du maître portugais Réis.

Mère ne savait que penser de l'absence de père mais elle était sûre d'une chose : Berg méritait bien d'être mort deux ans plus tôt, à l'âge de cinquante ans, après avoir eu le culot de composer un opéra sur des événements aussi honteux. Ce n'était que justice, gloussait-elle souvent et avec impudence, s'il n'avait pas eu le temps de terminer un troisième acte avant de mourir. Quelle époque terrible! Quelques mois plus tard, nous partions pour l'Amérique.

J. R. : Ne trouvez-vous pas ironique - bien que ce ne soit peut-être pas le mot juste vu ce que votre père a réalisé avec une chanson seulement - que précisément dans ce troisième acte de l'opéra, que nous ne connaissons que depuis 1979, Berg écrive des variations sur une humble chanson de cabaret composée par Wedekind lui-même? Je crois qu'Adorno dit quelque part que Berg décrit avec grossièreté, à l'aide d'une « musique déchue », la déchéance de Lulu. 
F. L. N. : Que de sottises prétentieuses ! Oui, je connais la référence : elle se trouve dans «Im Gedächtnis an Alban Berg " [Souvenirs d'Alban Berg]. [II se met soudain à chanter sur le ton d'un enfant qui geint :] «Berg et moi »"Moi et Alwan" [sic]. Il prétend que c'est lui, à l'âge de vingt-quatre ans, qui a orienté Berg vers les pièces de Wedekind avec de nombreux arguments convaincants. Berg, comme vous savez, envisageait également de composer un opéra d'après Und Pippa tanzt de [Gerhart] Hauptmann. Quel culot! Adorno dit rarement quoi que ce soit pour élucider ce mystère; il parle pour s'écouter parler. Tous ses écrits sur la musique me donnent cette impression. L'élément absolument primordial en ce qui touche la Lumpenballade [ballade populaire] de Wedekind est qu'il ne s'agit pas d'un cas isolé, car si c'en était un, Adorno aurait eu raison de lui accorder autant d'importance. Mais - Dieu nous en préserve ! - je pense que l'arbre lui cache la forêt, et que cet arbre se dresse tout seul au milieu de sa prairie stérile.

Berg a toujours utilisé des citations, sans exception, comme je l'ai démontré dans de nombreux articles, y compris ceux où je me livre à une polémique avec [le musicologue américain] George Perle. II le fait abondamment dans chaque œuvre. Parmi quelques exemples absolument étonnants :

- la mélodie Siehe, hier sind keine Menschen [Regardez, il n'y a personne ici] dans le dernier mouvement des Fünf Orchesterlieder, Opus 4, mieux connu sous le nom d'Altenberglieder. Les cartes postales écrites à la main par Peter Altenberg étaient conservés dans une collection privée mais, grâce à une relation dans l'aristocratie, Berg a eu la permission de les voir. C'est là qu'il a découvert la mélodie hexacordale (sans rythme) qu'Altenberg avait gribouillée en dessous de ces mots (Berg la transpose une tierce majeure plus haut; les notes originales d'Altenberg étaient inscrites sur une portée sans clef et sans lignes supplémentaires). Pourquoi Altenberg a attribué des notes à ces seules paroles est une question encore sans réponse. Perle ne fait que spéculer. Quant à Adorno, eh bien, il ne parle que de lui-même une fois de plus, dans la mesure où les Altenberglieder lui ont inspiré la description de diverses particularités dans le style de composition d'[Adrian] Leverkühn dans Doktor Faustus ;

- la berceuse Eipopeia dans Wozzeck, qui doit son origine à une photographie d'une partie des fragments d'un manuscrit de Büchner où ce dernier avait écrit, dans la marge, cinq notes sur une portée sans clef (Berg les lit dans la clé de sol). Berg avait obtenu cette photographie d'une aide-bibliothécaire particulièrement coopérante qui travaillait à la Staatsbibliotek de Munich vers 1917, en contrepartie d'un service spécial que Berg lui avait rendu.

J. R. : Peut-être pourrions-nous maintenant parler de votre nouvel ouvrage, Musical Compo.... 
F. L. N. : ... Excusez-moi, John, mais je dois dire que j'attendais ici une autre question au sujet d'Adorno. Je ne voudrais pas changer le cours de votre entrevue, mais... revenons-y plus tard, O.K. ? II y a d'autres choses que j'aimerais dire sur cette espèce de.... bouledogue!

J. R. : Très bien. C'est entendu. Votre livre Musical Compositions of the Century paraîtra sous peu. Félicitations. Le siècle est presque terminé. Je sais que vous venez de corriger les placards, et le peu que vous m'en avez dit laisse entrevoir un ouvrage fantastique. Un nombre surprenant d'œuvres canadiennes, m'avez-vous dit, figurent dans vos catalogues et commentaires. Pourriezvous résumer brièvement la thèse sur laquelle repose le livre? Vous pourriez peut-être aussi parler de ce qui vous a poussé à l'écrire.

F. L. N. : Très simplement, je me suis posé cette question: quelles sont les œuvres musicales qui ont défini, formé, marqué, donné un cadre à notre époque, au cours des cent dernières années - de 1895 à 1995 ? Les œuvres pivots qui ont influé sur le cours des événements musicaux - pour le meilleur et pour le pire ? Qui ont décrit de nouveaux univers sonores? Qui ont donné du plaisir à des milliers, que dis-je, à des millions d'auditeurs ? Je me pose des questions de ce genre depuis plus de vingt ans, surtout depuis que j'ai accepté le fait qu'il était trop tard pour renover avec mon passé de compositeur. Vous n'êtes pas sans savoir que je suis un auditeur avide et que je dévore les partitions, quand elles sont disponibles. C'est cette vaste expérience, et beaucoup de bonne volonté, qui m'ont incité à énumérer les 123 points cardinaux, les 123 œuvres clés. Je suis attiré par des œuvres de référence, pas par des compositeurs de référence. Je m'intéresse aux idées qui durent. Ars longa, vita brevis.

J. R. : Si je comprends bien, cela signifie que vous traitez aussi de la musique populaire?

F. L. N. : Oui, évidemment, mais seulement dans la mesure où il peut y avoir des idées dans la musique populaire. En outre, j'emploie douze catégories générales. Aimeriez-vous savoir lesquelles?

J. R. : Oui, s'il vous plaît. Et si nous avons le temps, pourriez-vous fournir un exemple de point cardinal dans chaque catégorie ? Je suis vraiment curieux de savoir comment vous êtes arrivé au chiffre 123 .

F. L. N. : Avec grand plaisir. Prenez cette photocopie que j'ai faite à votre intention; elle vous sera d'un grand secours. Les catégories sont: 1) Monuments du modernisme ; 2) La nature du son. Le son de la nature ; 3) Le progrès par la protestation; 4) L'arrivée des étrangers; 5) Exercices spirituels ; 6) Showbusiness et démocratisation ; 7) L'éternel féminin dans la (dé)cadence ; 8) Oligarchie et industrie du spectacle ; 9l Calamité, catastrophe et cataclysme ; 10) Ailleurs utopiques; 111 Complètement parti ; 12) Contes de fées. 
J. R. : [...] Extraordinaire! Je peux seulement vous dire que ces catégories, époustouflantes par leur originalité, ne me semblent pas d'une évidence limpide, du moins à première vue. Je remarque que, curieusement, vous n'utilisez pas le qualificatif très à la mode de postmoderne. Et, pour tout l'or au monde, je ne pourrais commencer à imaginer ce que vous entendez, par exemple, par la catégorie ; 4) L'arrivée des étrangers.

F. L. N. : Post-Mördern [post-meurtres], voilà ce que je dis. Logique énervée à la française, totalement insensée. Débilitante! [Jean-François] Lyotard ${ }^{(3)}$ et compagnie... Quel toupet! Ce terme ne signifie absolument rien pour moi. Et je crois que tout Musikwissenschaftler [musicologue] qui se laisse aller à l'utiliser se trompe. Tout ceci est en réalité une immense supercherie, une fraude pure et simple qui a cours depuis quelque temps, et je suis convaincu qu'une activité criminelle intense est associée à sa dissémination comme terme technique dans les universités du monde entier, aux États-Unis surtout.

J. R. : (... !) Je ne suis pas certain d'être d'accord, mais... peut-être pourrionsnous revenir à la catégorie 4), L'arrivée des étrangers. Auriez-vous un exemple pour $m^{\prime}$ aider à comprendre l'idée générale? S'il vous plaît ?

F. L. N. : Sans plus tarder ! Je vous citerai trois œuvres de référence, écrites presque au même moment dans l'histoire, et prouvant de manière très naturelle la présence d'un Zeitgeist [esprit de l'époque] : l'apparition d'une activité sportive, le tennis, sur la scène de concert dans le ballet Jeux de Debussy; I'arrivée d'une activité de bas étage, le cabaret, dans le mélodrame Pierrot lunaire de Schönberg; I'arrivée d'une concision tout orientale dans les Six Petites Pièces pour piano, opus 19, de Schönberg.

J. R. : (... ?) Je confesse avoir un peu de difficulté, disons, avec le rapport entre votre concept de l'idée et, pour utiliser un terme que vous accepterez sûrement, le concept de style. Ne pensez-vous pas, comme disait Schönberg, qu'il devrait y avoir un équilibre judicieux entre le style et l'idée, entre la manière et la matière?

F. L. N. : [Rire sonore et prolongé.] II fallait que vous choisissiez celui-là ! Rappelez-vous, fiston, que mon livre ne porte pas sur des gens, mais sur des œuvres. Schönberg a fait ce qui devait être fait : il a obligé l'idée à dominer le style. Par contre, dans ses œuvres de jeunesse - oh, quel terme inapproprié, car ce sont des chefs-d'œuvre entièrement formés, des œuvres irrésistibles d'une grande maturité - il avait permis au style de dominer l'idée. Et il en a éprouvé du remords. S'il a jamais équilibré les deux, votre manière et votre matière, il l'a fait dans quelques œuvres comme Pierrot lunaire, pas dans les Cinq Pièces pour orchestre, opus 16; dans les Pièces pour piano, opus 19, pas dans les pièces pour piano de l'opus 11 . Cette dichotomie est un sentier qui ne mène nulle part. Webern, c'est l'idée pure ; Berg, le style pur ; Schönberg, la confusion
(3) Niemantz fait référence aux écrits de Jean-François Lyotard, La Condition postmoderne (Paris, Éd. de Minuit, 1979) ; et aussi Le Postmoderne expliqué aux enfants (Paris, Éd. Galilée, 1988). En privé, Niemantz a également cité Roland Barthes, Jacques Derrida et Michel Foucault, entre autres. À cet égard, il est intéressant de noter que le philosophe allemand Jürgen Habermas associe le postmodernisme au néo-conservatisme français; voir son article " La modernité : un projet inachevé ", conférence traduite en français et publiée dans Critique, 413 , octobre 1981. [N.d.É.] 
pure. Laissez-moi vous rappeler que le rationalisme conduit directement au castrat !... et à... Buchenwald!

\section{J. R. : (... ! ?) Je... je ne sais trop que dire...}

F. L. N. : ... Adorno, ce vieux communiste qui n'est jamais sorti du placard, ne comprenait rien à l'Europe, ou du moins, il a fait semblant de ne rien comprendre. II a voulu nous faire avaler toutes ces notions excentriques sur les valeurs bourgeoises. Mais je suis convaincu que cela ne reflétait pas ses opinions véritables. II a adopté cette position bizarre pour vendre plus de livres, je suppose. Ce dont il aurait dû parler, c'est des valeurs aristocratiques, à la fois nobles et quasi royales, quelque chose que les Européens connaissent depuis des siècles; des valeurs aristocratiques dont les meilleurs artistes et compositeurs ont développé les implications dans leur cuvre. Prenez luigi Nono, par exemple. Comprenez-vous quelque chose à cela ? Le sentier emprunté par un autre compositeur vous semblera peut-être plus évident : quand Chopin... Non, non. Quand liszł compose sa Rhapsodie hongroise $n^{\circ} 2$, il " aristocratise " la musique tzigane, la musique folklorique dont elle s'inspire.

En Amérique du Nord, au Canada tout spécialement et surtout au Québec à cause de l'éternelle domination de l'Église, une alliance coupable de la démocratie avec les valeurs bourgeoises a virtuellement ruiné toute chance de créer une œuvre artistique digne de ce nom. La musique et la composition musicale, vous le savez mieux que moi, sont les premières choses qu' on laisse tomber quand les gouvernements élus font appel à la responsabilité fiscale, à la responsabilité bourgeoise. J'ai bien peur que, partout dans cet hémisphère, on soit en train de jeter le bébé avec l'eau du bain ! Franchement, vous méritez l'espèce de... [il cherche ses mots] musique de concert insipide et parfaitement niaise que l'on compose dans votre pays! Dieu du ciel! Et les commandes émanant de vos maisons d'opéra, de vos orchestres et formations de chambre : ils devraient faire autre chose que de cultiver une variété de mauvaises herbes aussi résistante! Chétives broussailles de prairies ! Où sont vos mécènes sérieux et bien informés? $N^{\prime} y$ a-til personne dans votre pays qui aime les jardins paysagés avec soin?

Nous avons essayé de remédier à cette situation, il y a de nombreuses années. Il fut un temps où je rendais régulièrement visite à Thomas von Hartmann ${ }^{(4)}$ tout près de Montréal, mais les circons...

J. R. : ... Est-ce que j'ai bien compris : vous avez rendu visite à l'assistant de [Georges Ivanovich] Gurdjieff(5) ¿̨ Au Québec?

F. L. N. : Oui. Je croyais que vous étiez au courant. Il lui arrivait fréquemment de passer ses vacances à Rawdon au début des années 1950, et je le voyais souvent, même si les déplacements en train n'étaient pas des plus agréables, surtout une fois du côté de la frontière canadienne. Mauvais rails, si je
(4) Thomas Alexandrovich de Hartmann. Niemantz "germanise " son nom à dessein. La famille de Hartmann appartenait à l'aristocratie russe en Ukraine, où il naquit en 1886 . Son grand-oncle, le philosophe Eduard von Hartmann, s'est rendu célèbre par sa Philosophie des Unbewußten [Philosophie de l'Inconscient]. À titre de compositeur, Hartmann a d'abord été lié à Kandinsky et à I'Almanach Der Blaue Reiter; il a signé l'essai «Sur l'anarchie en musique » ef créé la musique pour la pièce de Kandinsky Der gelbe Klang [le son jaune]. Hartmann et son épouse Olga furent des disciples de Gurdjieff (voir plus loin) pendant les années 1917-1929;

Olga fut la secrétaire de ce dernier. Tout au long de son association avec

Gurdjieff, Hartmann a travaillé avec lui à titre de co-compositeur et de co-arrangeur des mélodies que Gurdjieff se rappelait de sa jeunesse et de ses voyages, et qu'il utilisa plus tard dans son enseignement, soit sous forme de mouvements de ballet, soit à des fins de méditation. À partir de 1922, ils vécurent en France où Hartmann écrivit la bande sonore de plus de 50 films. En 1950, ils immigrèrent en Amérique. Hartmann mourut à Princeton en 1956. Son autobiographie posthume s'intitule Our Life with Mr. Gurdjieff. [N.d.É.] (5) Professeur et leader du mouvement spirituel qui porte son nom, Georges Ivanovich Gurdjieff est né en Russie en 1872 dans une famille d'ascendance grecque et arménienne. Ayant étudié plus de deux cents religions, il voyagea dans toute l'Asie "à la recherche de la vérité "; le récit de son périple figure dans un ouvrage Rencontres avec des hommes remarquables. Son Institut pour le développement harmonieux de I'homme déménagea de Tiflis à Fontainebleau, près de Paris, en 1922. Parmi les nombreuses personnalités qui gravitèrent autour de lui, citons l'écrivain Katherine Mansfield, I'architecte Frank Lloyd Wright, le compositeur de musique électroacoustique Pierre Schaeffer ainsi que le metteur en scène et réalisateur Peter Brook. Gurdijeff mourut en 1949. [N.d.É.] 
me souviens bien. Quoi qu'il en soit, Hartmann, ses associés et moi-même avons tenté de concevoir et de proposer de nouvelles façons de vaincre les restrictions innombrables qui émergeaient dans la société québécoise, en abordant d'une manière aristocratique tous les aspects de la vie, à l'aide de la musique elle-même, de la formation et de l'interprétation musicales. Malheureusement, son état de santé laissait à désirer et nous avons dû abandonner nos projets pendant un certain temps.

J. R. : Tout ceci a quelque chose à voir, je suppose, avec votre conférence au congrès international de la STAVA ?

F. L. N. : Oui, jusqu'à un certain point. L'événement a lieu à Montréal en son honneur. Les problèmes au Québec, au Canada et dans les Amériques ont atteint des proportions inimaginables, et si l'art doit survivre, si l'esprit doit continuer son ascension... [Pause.] Pardonnez-moi... Nous nous éloignons de notre sujet, $i^{\prime}$ en ai bien peur. Je préférerais retourner à notre conversation sur les notions contenues dans mon nouveau livre.

J. R. : Bien sûr. Avec plaisir.

F. L. N. : En identifiant les œuvres musicales clés de ce siècle, j'aurai contribué de façon modeste mais néanmoins importante à accélérer la quête de vérité musicale chez les jeunes. Ils n'auront plus besoin de cheminer péniblement et sans résultat à travers une autre... une autre vaste prairie de mauvaises herbes broussailleuses et vénéneuses.

J. R. : Formidable ! Nous parlons des douze catégories. Auriez-vous une objection à passer chacune d'entre elles rapidement en revue - sans révéler chaque œuvre clé naturellement, après tout on veut que les gens achètent votre livre - et à donner à nos lecteurs un aperçu de votre proposition ? Le nom de l'œuvre suffira, je pense, le nom du compositeur peut être facultatif, mais la raison qui a motivé votre choix serait très utile, si vous voulez bien la partager avec nous.

F. L. N. : Excellente suggestion. Cela nous ramènera à nos moutons. Allons-y.

J. R. : Catégorie 1): Monuments du modernisme?

F. L. N. : Les Fünf Klavierstücke, opus 23 [de Schönberg] indiquent un "nouveau champ "; Musique pour cordes, percussion et célesta [de Bartók] révèle une "nouvelle géométrie "; la Symphonie, opus 21 [de Webern] nous enseigne que "less is more "; la Sinfonia concertante pour tuba, clarinette basse, piano et orchestre de Hindemith nous enseigne que "more is less"; Pulcinella [de Stravinski] utilise "le passé comme présent »; je distingue également dans cette catégorie: "le passé comme futur"; "le présent comme passé "; "le présent comme futur "; " le futur comme présent »; "le futur comme passé ». 
J. R. : (... ?) Catégorie 12): Contes de fées?

F. L. N. : Carmina Burana [d'Orff] et Einstein on the Beach [de Glass], musiques dont les enfants se souviennent pour la vie parce qu'ils ont eu peur à la première audition et qu'ensuite, devenus adultes, ils désirent y retourner encore et encore et encore et encore et encore et encore et encore et encore et encore et encore et encore et encore et encore.

J. R. : (... !) Catégorie 2) : La nature du son - Le son de la nature?

F. L. N. : Gesang der Jünglinge [de Stockhausen], la composition électroacoustique annonciatrice qui a fait l'effet d'une molécule d'ADN agressive dans le milieu des compositeurs du monde entier; The Princess of the Stars [du compositeur canadien R. Murray Schafer], une Water Music de notre époque à l'intention, comme des critiques l'ont prétendu, des écureuils, des porcs-épics et des oiseaux migrateurs.

J. R. : (... ?) Catégorie 11): Complètement parti ?

F. L. N. : Purple Haze [de Jimi Hendriks].

J. R. : (... !) Catégorie 3) : Le progrès par la protestation?

F. L. N. : Amériques [de Varèse] et The Steel Foundry [de Mossolov].

J. R. : (..) Catégorie 10) : Ailleurs utopiques?

F. L. N. : Gruppen [de Stockhausen] et Répons [de Boulez].

J. R. : (...) Nous avons déjà vu 4), alors voyons : la catégorie 9) : Calamité, catastrophe et cataclysme.

F. L. N. : Des œuvres merveilleuses de lannis Xenakis : Metastasis, Pithoprakta, Terretektorh, Kottos, Antikthon, Akrata, Dikhthas, Ikhoor. Foxy Lady [de Jimi Hendriks] figure également dans cette catégorie.

J. R. : (... ! ?) Catégorie 6) : Showbusiness et démocratisation?

F. L. N. : La Bohème, Tosca.

J. R. : (... ?) Catégorie 8): Oligarchie et industrie du spectacle?

F. L. N. : Licht, l'opéra de sept jours de Karlheinz Stockhausen qui sera présenté intégralement en 2008 pour célébrer le quatre-vingtième anniversaire de naissance de son créateur; aussi : Le Fantôme de l'Opéra [d'Andrew Lloyd Webber]. On trouve également ici une de mes œuvres préférées: Bad de Michael Jackson. Sans l'édifice de soutien oligarchique que nécessitent ces spectacles à grand déploiement, il n'y aurait aucune place pour l'art grandiose dans notre monde. 
J. R. : (... !) Catégorie 7) : L'éternel Féminin dans la (dé)cadence ?

F. L. N. : Le Boléro de Ravel, et cette autre inspiration étonnante, Pulau Dewata du Montréalais Claude Vivier. Je n'ai cité aucun enregistrement sur $C D$ de la première œuvre ou des diverses orchestrations de la seconde. Mais je sais que les Montréalais sont très fiers des enregistrements de leur Orchestre symphonique, et tout autant de votre orchestration très sensible du chef-d'œuvre de Vivier.

J. R. : (... ! ? ! ?) Merci, merci beaucoup. (... ? ! ? !) Nous arrivons maintenant à la dernière catégorie, la catégorie 5) Exercices spirituels. Que peut bien contenir cette case?

F. L. N.: Harmonielehre, de John Adams, et le Quatuor à cordes no 2 [durée : quatre heures trente] de l'inimitable Morton Feldman.

J. R. : Vraiment ! Comment cela ?

F. L. N. : Les œuvres dans cette catégorie lil y en a aussi un certain nombre issues du free jazz, du rock acide, du disco et des styles chrétiens charismatiques contemporains) tentent de maintenir un lien étroit et intime avec l'auditeur, lequel est tacitement invité à retarder ses applaudissements. Et, comme c'est le cas pour la musique de Bach, Haydn, Mozart, Beethoven, Spohr, Wagner, Bruckner, Brahms, Humperdinck, Strauss, Pfitzner et Mahler, plus on retarde le besoin d'applaudir, plus la détente orgasmique est grande lorsque les applaudissements arrivent enfin. Pendant plus de deux cents ans, toute la tradition austro-germanique de l'opéra et de la symphonie s'est appliquée à exercer ce principe spirituel, le principe du sexe tantrique. D'autres ont tenté de l'imiter, évidemment, et avec raison.

Les Italiens sont multi-orgasmiques, de toute évidence, comme le confirmera n'importe quelle soirée dans une loge d'opéra. Nos frères et sœurs latins interrompent chaque air d'applaudissements impétueux, ponctués de nombreux " ah", "pas trop vite " et ancora, ancora. Mais cette exubérance leur coûte cher : il n'y a jamais eu - et il n'y aura jamais - de tradition symphonique en Italie.

Les Français, dans leurs bons jours, ont un peu du Nord et une touche du Sud. Avez-vous remarqué récemment que Boulez a entrepris de réviser plusieurs de ses œeuvres, toujours en les... allongeant? Berio adore folâtrer. Ligeti : aberatio mentalis partialis. Chez Stockhausen, aucun doute : Licht doit être placée dans l'empyrée des exercices spirituels, seule œuvre dans mon ouvrage à figurer dans deux catégories distinctes !

Les Anglais, naturellement, ne possèdent de tradition ni dans la symphonie ni dans I'opéra. La répression totale! « Pas de sexe, s'il vous plaît, nous sommes [il rit à gorge déployée] britanniques. » 
J. R. : Si j'ose demander, qu'avez-vous à dire sur... le Canada?

F. L. N. : [...]

J. R. : ... Je veux dire le Québec?

F. L. N. : Vos compatriotes jouent avec les principes socialistes depuis trop longtemps; il est temps de changer. Ces valeurs ne servent plus à rien. Tout comme le port de masques protecteurs au hockey a entraîné un nombre record de blessures, tout comme les coussins gonflables ont augmenté le nombre $d^{\prime}$ accidents de voiture mortels, les règlements sur le contenu canadien ont contribué à la création de milliers d' "enfants morts-nés ", même dans le domaine de la composition, bien sûr. Tant de musique morte ! Tant de blessures!

Vos compatriotes écoutent Beethoven un peu comme ils regardent la télévision : c'est une expérience par procuration, asexuée, où toutes les batailles qu'on voit et qu'on entend - dans la musique - ont déjà été gagnées et déployées avec soin entre des pauses publicitaires. Voilà pourquoi tant de vos compositeurs capitulent, préférant ne pas toucher ou provoquer leurs auditeurs, préférant ne pas composer de la musique mais... réécrire ce qui a déjà été apprivoisé, éviscéré. Musique sans trace d'exercice spirituel. Presque toute la musique composée dans votre pays est du genre conçu pour convection rapide, comme si elle était faite pour être réchauffée au micro-ondes. Vingt minutes de répétition et ça y est : on donne le concert! C'est une musique sans mécène!

\section{J. R. : $(\ldots)$}

F. L. N. : Récemment, au sein de notre mouvement, un groupe de disciples de Hartmann et moi-même nous sommes battus contre une faction canadienne dissidente qui proposait très sérieusement lau point d'en faire une priorité dans nos démarches) le plan suivant: les fonds accordés aux conseils des arts par vos gouvernements à l'intention des compagnies d'opéra et des orchestres symphoniques seraient réacheminés au gouvernement fédéral pour que ces formations musicales soient subventionnées directement par Santé et Bien-Être Canada, compte tenu de tous les bienfaits médicaux qu'elles apportent à leurs publics en leur fournissant une proportion extrêmement réduite d' $d^{\prime}$ enfants morts-nés » dans le domaine de la musique "vivante ", ainsi qu'en les approvisionnant avec régularité en compositions digestibles.

On avait même proposé une forme de taxation visant à obliger tous les contribuables ayant un emploi à verser un montant perçu chaque mois sur leur chèque de paie. Ce système devait s'appeler, dans la version anglaise, "Music for All/All for Music ». La version française devait s'intituler « Musique à l'heure/ Alors la musique ». Dans les deux cas, l'acronyme était MAAM. Par exemple, quiconque assistait à un nombre $x$ de concerts ou de représentations d'opéra 
au cours de l'année pouvait déclarer la déduction MAAM dans sa déclaration de revenus. L'objectif de ce programme était sans doute honorable, puisqu'il aspirait à augmenter l'assistance au concert et à l'opéra. Mais l'un de nos associés de Trinidad s'est objecté, alléguant que, prononcée incorrectement, l'abréviation pourrait favoriser les conflits raciaux. Quels zélotes mal inspirés ! Ils essayaient probablement de comprendre et de mettre en pratique les écrits socialistes emberlificotés de Wiesengrund. Quelle farce!

L'assurance-maladie, c'est-à-dire l'accès universel au système de santé, ne fonctionne pas; les règlements sur le contenu canadien non plus. Les riches sont toujours allés ailleurs chercher leurs "soins", chercher leur " art " et leur "musique ». Un mécène est un citoyen très consciencieux. C'est un élément normal de l'ancien principe aristocratique.

Par la faute de vos propres règlements, vous avez déprécié votre propre culture, votre propre société. Vous avez réussi votre dictature du prolétariat! Mais à quel prix?

Les Canadiens n'ont jamais connu le divin en musique ; les Québécois ont oublié ce qu'ils savaient. Vivier avait un petit peu, un tout petit peu, juste un peu de sacré. Quoi qu'en disent les anticréationnistes sur la primauté de la nature, la nature de la musique ef la musique des oiseaux (pauvre Aristophane, paix à tes cendres!!), la société passe en premier, l'art en second. Les temps de la musique sont malsonnants parce que les tempi de la société sont malséants. La société doit tenter de fournir à ses enfants l'éducation élégante que les aristocrates ont toujours donnée aux leurs: ce qu'il y a de mieux! Réparez votre société !

\section{J. R. : $(\ldots)$}

F. L. N. : (...)

J. R. : Cela me ramène... au temps de Princeton, dans votre classe. Incroyable. On oublie parfois. On oublie les choses les plus importantes. J'avais oublié votre mot préféré : aristos [le meilleur]. J'avais oublié ce qui m'opposait à vous de façon si véhémente à l'époque. Je me rends compte maintenant que je n'avais même pas compris votre autre observation révélatrice: "C'est le pire de tous les mondes possibles. »

F. L. N. : Oui, c'est tellement vrai. Tous sont allés cultiver leur petit jardin... seuls. Une chose est sûre : la plupart ont oublié les mauvaises herbes.

J. R. : Avant de conclure aujourd'hui, je ne peux passer sous silence le fait que vous célébrez demain votre soixante et onzième anniversaire de naissance. Mes meilleurs souhaits. 
F. L. N. : Merci. Merci beaucoup. C'est très gentil à vous de vous en souvenir. Oui, je suis né dans une bonne décennie. Le destin prodigue parfois à l'humanité un rayon d'espoir. 


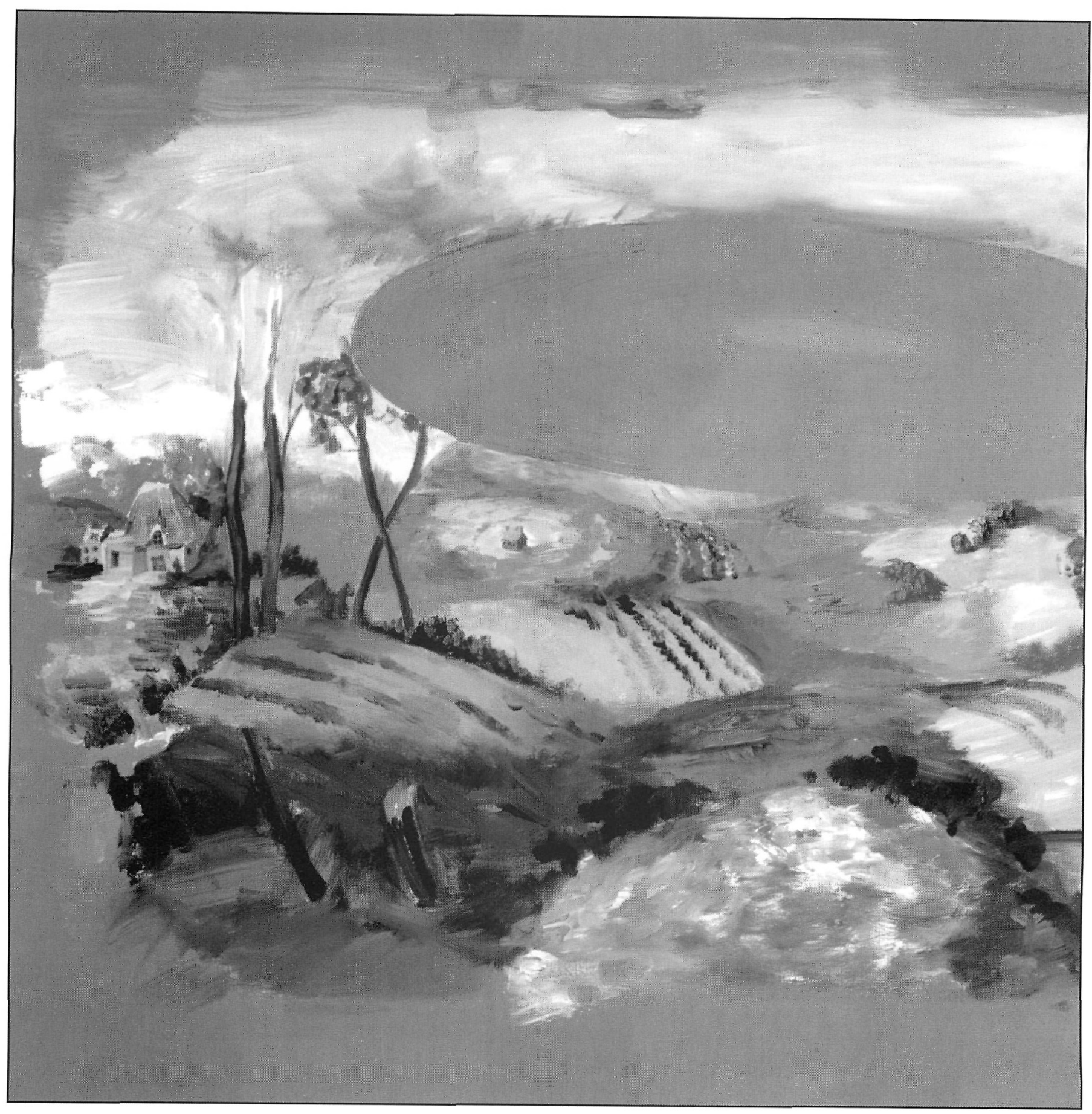

